$\left(25^{\circ}-32^{\circ} \mathrm{C}\right.$.) or relative humidity $(64 \%-84 \%)$. Because of the close correlation between these two variables, it was not possible to separate their effects on the eclampsia rate. Direct account could not be taken of domiciliary confinements in the city, though there was reason to believe that all eclamptic women were admitted to hospital.

It could be argued, perhaps, that cool or humid days might persuade some normal women to stay at home to deliver while those with eclampsia would continue to enter hospital-thus creating the impression in hospital that the eclampsia rate was higher on such days. However, this effect of coolness and humidity was considered to be real, since even a mild degree of coolness had an appreciable effect on the eclampsia rate, whereas rainfall had none. The absence of any significant correlation between humidity or temperature and blood pressure or oedema suggests that the effect was not mediated by vasospasm or fluid retention. Moreover the effect of cool weather was too large to be explained by a reduction in sweating, with a consequent increased likelihood of eclampsia through persistence of fluid retention. The nature of the connexion between eclampsia and the weather therefore remains obscure.

The immediate public health implications of the findings are minimal, but clearly it would be of interest to see if the relationship can be confirmed in other parts of the world. Meanwhile, it seems that eclampsia may have to be added to that varied group of conditions which are affected by the weather: which in addition to respiratory infections, includes ischaemic heart disease, peripheral vascular disease, systemic lupus erythematosus, and arthralgia-not to mention emotional states.

1 Neutra, R., Fournal of Obstetrics and Gynaecology of the British Commonwealth, 1974, 81, 833.

\section{Management of Recurrent Head and Neck Cancer}

The reappearance of a neoplasm following potentially curative therapy by radiation or surgery is conventionally described as a "recurrence," whether it is residual tumour left after ineffective resection, regional metastasis, or regrowth at the initial site after radiotherapy. Some tumour areas in the head and neck carry a markedly better prognosis than others, so overall recurrence rate will vary with the total experience of the surgeon as well as the distribution of the tumours he is treating. Despite the failure of the initial treatment, some patients can still be cured of their neoplasms; others can be offered only palliation of varying duration and effectiveness. The choice of this second line therapy and its successful accomplishment require considerable experience and adequate supporting facilities if the patient's sufferings are not to be increased. This must of necessity mean a degree of specialization, for such knowledge can be gained only by the care of large numbers of patients.

Inadequate surgical excision of the original tumour can rarely be rectified by a second operation. Radiotherapy has little to offer in the enhancement of cure rates in these patients but may have some palliative value, particularly the increasingly popular fast-neutron therapy.

Williams ${ }^{1}$ has recently emphasized the varying salvage rates for recurrent tumours in differing sites of the head and neck, and though his personal series was small-144 patients treated in six years (about the number seen by most large centres in less than a year)-his conclusions were apt. Tumours arising in areas inaccessible to radical excision are rarely cured if they recur after initial therapy-usually radiotherapy alone or in combination with an attempted radical excision. Nasopharyngeal carcinoma and tumours of the middle ear, base of tongue, and paranasal sinus are examples of this problem. On the other hand, secondary total laryngectomy or the technically more demanding pharyngolaryngoesophagectomy yields a generous proportion of long-term cures if carried out adequately and early in the recurrence-it is sometimes forgotten that there are degrees of recurrence and technical limitations to even the most enthusiastic surgeon's expertise. Extension to the skin and the need for its replacement require knowledge of the proper techniques of reconstruction, for there are inherent difficulties in the application of standard plastic procedures to previously irradiated tissues.

The problems in carrying out salvage procedures for tumours recurring in the oral cavity, where radiotherapy is invariably used as the initial mode of treatment, have been discussed recently by Harrison. ${ }^{2}$ Adequate excision is often possible, though it is of limited value if unaccompanied by effective rehabilitation of swallowing. Total glossectomy is a formidable procedure, even for young patients, while replacement of the whole or part of the mandible may be impossible after radiotherapy and suggests a need for urgent reappraisal of the initial therapy in these tumours.

Recurrence rates always reflect not only the effectiveness of the initial treatment but the incidence at sites, such as upper jaw and middle ear, which carry a very poor prognosis. Undoubtedly a combination of well-planned and carefully executed salvage surgery will result in the cure of some patients with recurrent disease arising at an advantageous site. Invariably the price to be paid will be high, not merely in money but, more important, in time and hardship. In the frail and the elderly this price may be too much. Perhaps a more rewarding approach would be better planned and executed primary treatment.

1 Williams, R. G., British fournal of Surgery, 1974, 61, 691.

${ }^{2}$ Harrison, D. F. N., Proceedings of the Royal Society of Medicine, 1974, 671 601 .

\section{Open Minds for Open Medicine}

Campaigning is under way for the Open University to start a medical faculty, in which mature students would pursue a part-time course at their own pace for some years and then, after passing examinations, become full time for their clinical training. Their medical qualifications would be no different from those granted by the existing medical schools in Britain.

On the face of it this seems an attractive proposition-a welcome supply of home-grown, dedicated doctors, often with previously learned skills, perhaps more interested in care than cure, produced at little expense; frustrated citizens turned, after character-proving struggle, to fulfilment; and a new use for the Open University's technical virtuosity. These were some of the benefits claimed at a conference on "Open Medicine and its Implications" held last weekend in Liverpool and organized by the Open Medicine Trust. ${ }^{1}$

Many people at the conference clearly thought that an Open University medical faculty was technically possible; but there 
was no unanimity that it was either necessary or desirable. Attitudes were still fluid, however, so that the participants were able to discuss the wide range of ideas expressed dispassionately. Are more doctors needed? Or should our present ones be used more effectively, particularly as up to $£ 1$ million may be channelled through the total career of each one ? Could the shortage be overcome by better distribution? Starting now, the Open University might produce 300-600 doctors a year from 1985. Would that make much difference, or would conventional medical faculties be producing too many doctors by then ? Reliable facts and forecasts are hard to come by-as Willink and Todd found in the past. Nevertheless, capital costs would be small, though revenue expenditure could be high.

The merits of the project had to be assessed more on opinion than on fact. But student views strongly supported the need to widen the discussion to include the health professions as a whole. It was desirable, and probably politically essential, that the members of the health team should not be treated separately, and that-with trade union help, enough money, and a broadly based entry-open medicine could pioneer a more realistic approach to health care. But how should the Open University choose its students? Was it wise to encourage students from the paramedical professions, already critically understaffed ? Should students with particular skills be chosen, or those who demonstrably cared for people?

This question, like many others, exposed deficiencies in the existing practice of medical schools-they had no clear criteria for selecting people who will make good doctors. The ever-increasing demand for higher A-level grades might be proving counterproductive-perhaps the cleverest were not the best choice. But were mature students any better ? Experience of the $5 \%$ already coming in annually was not wholly encouraging, though some were superb.

Techniques recently developed for existing Open University courses could be used to produce excellent preclinical tuition, but a large query hung over the clinical training. Modern medical educators prefer to introduce clinical contact in a wedge-shaped fashion from the start and to blur the old preclinical/clinical distinctions. For the Open University a preclinical degree course in medical sciences would be more practicable, and could be opened to other health professionals. But opinions on clinical training varied from short bridging courses in existing medical schools for groups of 50 students, followed by study in clinical education centres in district hospitals, to arrangements for interested consultants in 100 suitable district centres each to take six students a year.

Another view was that medical teaching had reached a crossroads. Because the pay had been poor for years, doctors had not been going into the preclinical disciplines to teach; and, now that junior academic staff could not earn as much as their contemporaries in the N.H.S., recruitment to clinical teaching was also falling off. Meanwhile student numbers had been increased. Would standards fall, or could they be saved by applying Open University techniques, not to an open medicine faculty but in the existing medical schools themselves?

The recently accepted E.E.C. requirement for 5500 hours' formal teaching in medicine, the need for G.M.C. approval of medical degrees, the need for money from the hard-pressed Government departments, and the Open University's own policy not to start new faculties add up to make the chances of open medicine, or even open health sciences, remote. Nevertheless, the crisis in the medical schools, stagnation in medical educational methods, and the inefficiency of much expensive health care, as well as the Open University's dramatic successes, together present a convincing case for investigation of these new approaches. If the medical and non-medical enthusiasts for open medicine could refine the multitude of ideas and sort out the facts about curricula cost, need, desirability, and criteria for selecting students, they might still not end up with an Open University medical faculty-but they might rejuvenate British medical education and ultimately medical practice.

1 Further details from The Open Medicine Trust, 36 Rudloc Road, London SW12 ODJ. Telephone: 01-675 1286.

\section{Genetics and Mental Retardation}

Several rare X-linked forms of mental retardation are recognizable by specific physical features-for example, the Hunter, Borjeson, Menkes, Norrie, and Lowe syndromes. In addition individual families have been reported with an obviously X-linked pattern of patients affected by moderately severe mental retardation, but with no specific features. This condition is often called Renpenning's syndrome after the reports in 1962 and 1963 of Canadian families by Renpenning and his colleagues. ${ }^{2}$ An earlier report was that of Martin and Bell $^{3}$ in 1943 of a family with 11 affected males in two generations and also two less seriously retarded females who might have represented heterozygote manifestation of the gene concerned.

Recently evidence has been accumulating that this nonspecific X-linked form of mental retardation is relatively common. Last year Lehrke ${ }^{4}$ described three large and two small pedigrees of patients from Minnesota showing an X-linked pattern. The I.Q.s of the affected members of these families ranged from 20 to 70, and in some instances at least the verbal I.Q. was lower than the performance I.Q. In 1973 Davison, ${ }^{5}$ who had searched for all families in the Oxford region in which there were two or more "severely subnormal" patients without specific diagnosis of whom at least one was still alive, reported finding 141 such families. There were 50 families with only males affected, 20 with only females affected, and 71 with both sexes affected. In eight of the families with only male patients there was evidence beyond the sibship of the index patient of X-linked inheritance. It may be presumed that in other families X-linked inheritance was responsible without this being clearly indicated by the family tree. Last year Turner and Turner ${ }^{6}$ from Sydney studied all schoolchildren with moderate retardation (I.Q.s of 30-55) born in 1955-64 and noted which of them had a similarly retarded brother or sister. They found 58 male patients with an affected brother compared with only 22 female patients with an affected sister. They were not able to look further for evidence of X-linkage in the families of the male patients with an affected brother; but in an earlier study ${ }^{7}$ of 14 men in institutions in New South Wales with non-specific mental retardation and whose families were available for study they had found indications of X-linked inheritance in seven.

The Turners suggested that almost all the excess of male sib-pairs in their recent study can be attributed to the X-linked form of mental retardation; and from this (perhaps questionable) assumption they estimate that this condition, or group of conditions, is responsible for one-fifth of all mental retardation 